\title{
A Novel Developed Linear and Nonlinear System Identification for an Industrial Dryer
}

\author{
Mostafa Darvishi \\ Department of Electrical Engineering \\ École Polytechnique de Montreal
}

QC, CANADA

\begin{abstract}
Dryer system is a controlled plant which is normally used in chemical industry. The proper modeling of this system facilitates its maintenance and keeping. Normally this is not an easy task, unless having a complete model of the process, due to sudden and nonlinear events. In this paper the linear identification of dryer plant in Arak petroleum, is introduced according to the Autoregressive with Exogenous Input (ARX) model and Box-Jenkins model and also the nonlinear identification based on Multi-Layer Perceptron (MLP) algorithm is investigated. The simulation results were satisfactory. It was concluded that these models can be used to design adaptive or robust controllers.
\end{abstract}

\section{Keywords}

Dryer, Linear system identification, Nonlinear system identification, ARX structure, Box-Jenkins model.

\section{INTRODUCTION}

A dryer system is an important control plant which can be widely explored for teaching control engineering. The dryer system is suitable as a system control example because it is very simple to understand and there are still various control techniques to be studied covering many important classic and modern design methods.
In the field of system identification, some recent works and ideas were explored to represent a suitable model which is more close to dryer system configuration. Identification of real industrial systems under load and under operation is a challenging task. Reviewing the other activities done in the field of physical system identification showed that the identification task was done in off-line mode with no system operation. This tendency could not be achievable for dryer system, because this industrial system could not be worked in off-line mode since its outputs were fed to other control parts. So, the novelty in this identification task was the formulization of some fundamental identification methods to realize dynamics recognition of this on-line system based on input-output system configuration and relation. By assuming the heater supply voltage as input and the heater output air temperature as output, the important feature of this SingleInput Single-Output (SISO) system is its stable open loop. Figure 1 shows the schematic of the dryer under investigation $[1,2]$. The system is a plant in Arak petroleum. It includes several parts such as motor, air supply fan, heater, dryer, cyclone unit, etc. The motor shaft has been connected to the air supply fan. Running the motor makes air supply that is blew to the heater. Figure 2 shows the input and output variables in this system. The step response for the dryer where the heater supply voltage is an input and the air temperature is output is shown in figure 3. As can be seen, in open loop case, the system is stable.

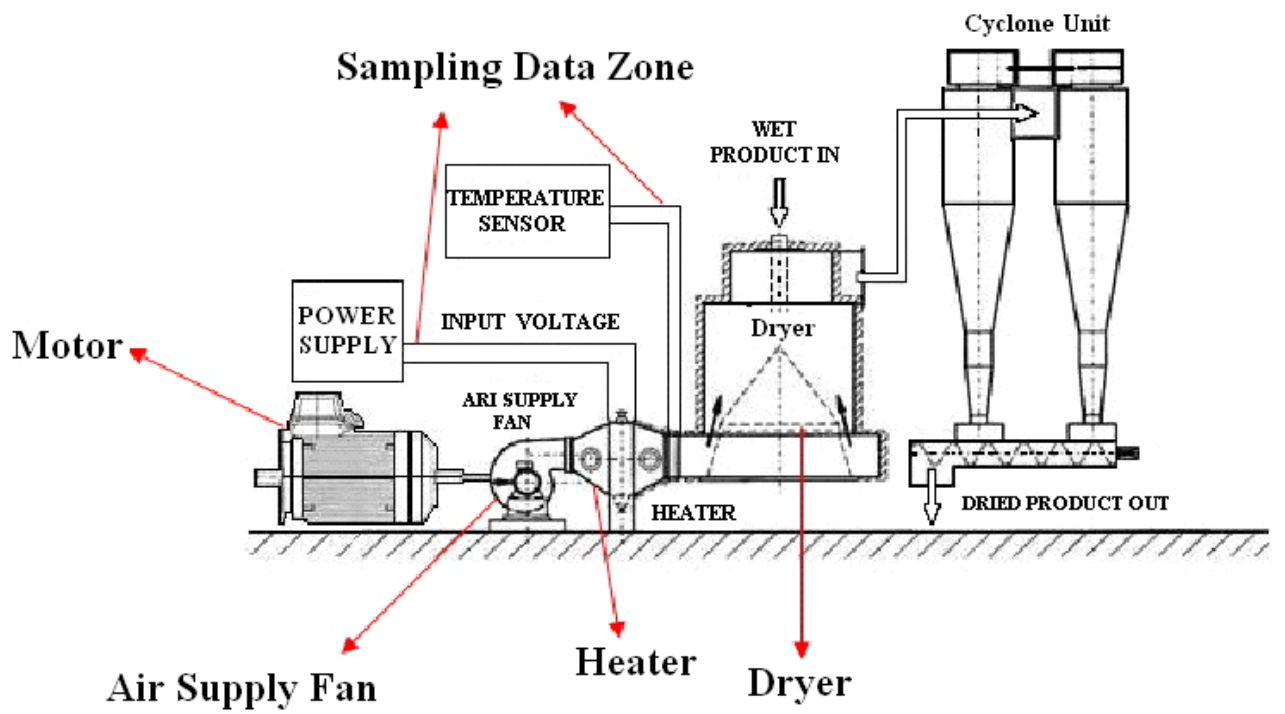

Fig. 1. A schematic of the dryer plant 


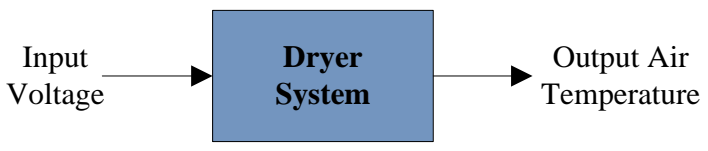

Fig. 2. Block diagram of dryer system based on inputoutput signals

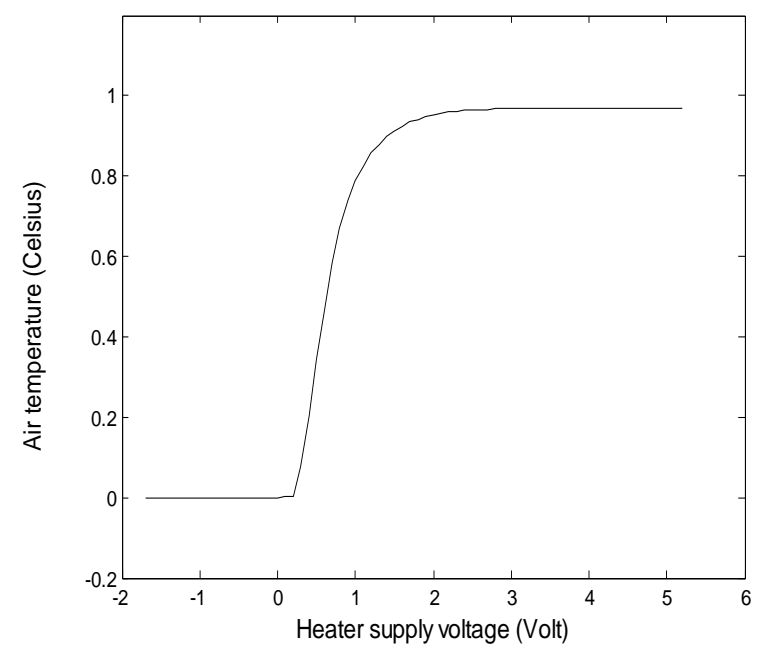

Fig. 3. Step response of dryer

\section{DATA ACQUISITION CONDITIONS}

There are three factors in modeling based on the identification techniques:

- Useful and valid information

- A perfect and useful model

- Strong method to adjust the model

According to the input and output data to recognize the system behavior, the output change must be touched from input. So, if the disturbance be low, the model validation will perform better. To meet this goal, the system must be stable before collecting data and any change should not occur in output.

The stage of data acquisition from dryer system was one of main challenges during this project. Since the system could not work on off-line mode and also its structure did not allow to follow the other related research manners, so this system was modeled as a Single-Input-Single-Output (SISO). To have a SISO system, the air temperature which was blown to the dryer having varied heater supply voltage was measured. A reason in choosing the supply voltage as input is because of its observable effect on the air temperature. Also, system identification just based on the input and output products, without considering the input air temperature, did not have a satisfactory result about model dynamics [1].

In comparison with the other related researches in identification mode, since this system was an industrial online sample, the direct use of identification methods could not comprise reasonable results. At the first time, some input and output data were collected by using two general sensors at input and output and tried to follow the methods which were done by other researchers [4,7], but no reasonable results obtained. Continuing research to overcome this problem, first, some precise sensors were located at system input and output for data acquisition, second, tried to do a novelty and formulize the fundamental identification techniques based on dryer system configuration, behavior, its initial delay and operation which achieved via some consultant and meetings with some engineers operating with this systems during recent years. The re-formulization results for this system to carry out the identification task will be represented in section 4 .

As a comparison between this identification method and other activities have been done by other researchers, this work had some advantages and disadvantages [5, 7]. The on-line system operation and also high temperature around the data acquisition points and some impurities such as dust and environment noise forced the use of precise sensors with higher sensitivities which were more costly. The advantages of this work are achieving identification task under load while system full operation, overcoming the environment undefined noise and distribution affecting sensors response by formulization of identification methods and realizing them by correct definition of system training via neural networks [11, 12, 14-16]. The disadvantage of this work is the influence of environment conditions such as high temperature around data acquisition points, dust and noise on sensors accuracy in input and output measurement in short times and the necessity of recalibration or treatment after some months. Actually, the industrial limitations for this system forced these conditions to the identification task $[8,13]$. Two types of sensors have been used in this plant. A voltage sensor for sampling the heater input voltage, and a temperature sensor for sampling the heater output air temperature. Both sensors sampled with $10 \mathrm{~Hz}$ rate $[9,30]$.

Data pretreatment, such as DC level correction and peak shaving, is a very important technique for better understanding of the process behavior. So the obtained data should have valid information to be usable for identification to make the process dynamics visible which is achieved by filtering and data pretreatment $[10,17,18]$. An important point about data acquisition to be considered is that the disturbances and unexpected events such as fan speed reduction and noise in the input voltage should not affect the system behavior. The dryer system identification is a passive task. So it is impossible for the model to have the same behavior with the real system unless satisfying these conditions in the model.

Figure 4 shows the change of the input (voltage) and output (temperature) signals. The voltage signal is analogue while temperature is given by a signal based on a Pulse Width Modulation (PWM).

\section{DATA PRETREATMENT}

The data from dryer contain high frequency noises and spikes. Sometimes immeasurable disturbances occur and take the system out of its linear range. Operation point change causes entering nonlinear effects in output data [2].

It is advisable to use a sampling frequency that is a multiple of the desired working frequency. This will build redundancy into the measured data that can reduce the information loss in the pretreatment of the data. When the data are acquired, one must always first plot the data in order to inspect them for these deficiencies $[19,31]$.

To solve some of these problems, some pre-processing methods mentioned in identification references were applied [3], to reach an optimized model of process. Thus, the effort was to choose a better model structure and focus on its flexibilities. 

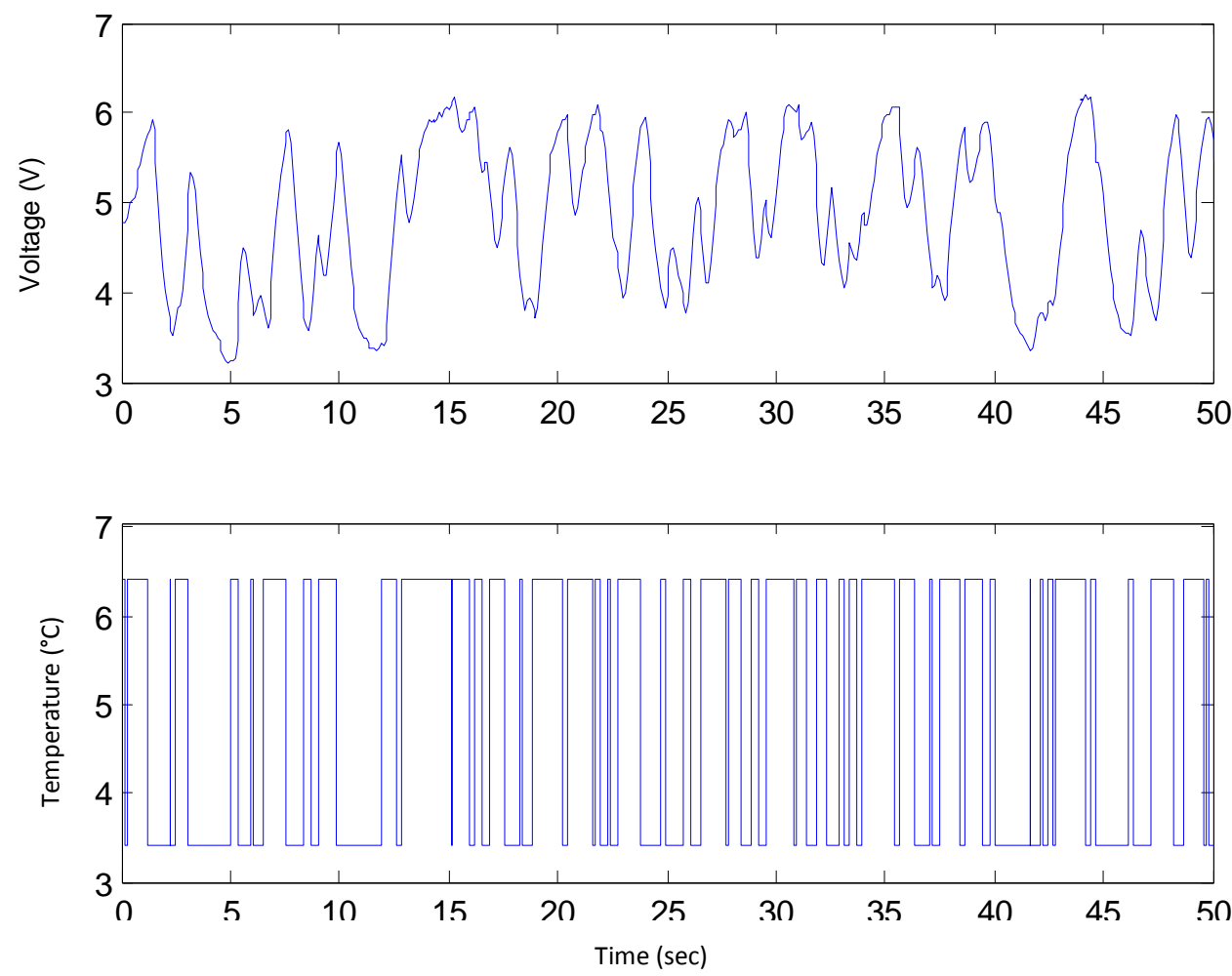

Fig. 4. Input and output data representation for dryer identification

\subsection{Level Correction}

In industrial practice all inputs and outputs have not the same order of magnitude. The obtained numerical values are related to physical quantities generally; do not have the same dimensions. The signals with the largest numerical values will automatically get the highest weight in the quadratic loss function which is minimized for determining the model, if these signals are directly used [32].

Notice that with DC level correction, the real level of data will be lost. When the system has a net integrator or it is unstable, analyzing the system without de level correction of data is not recommended. Figure 5 shows the DC level correction for input data [36].

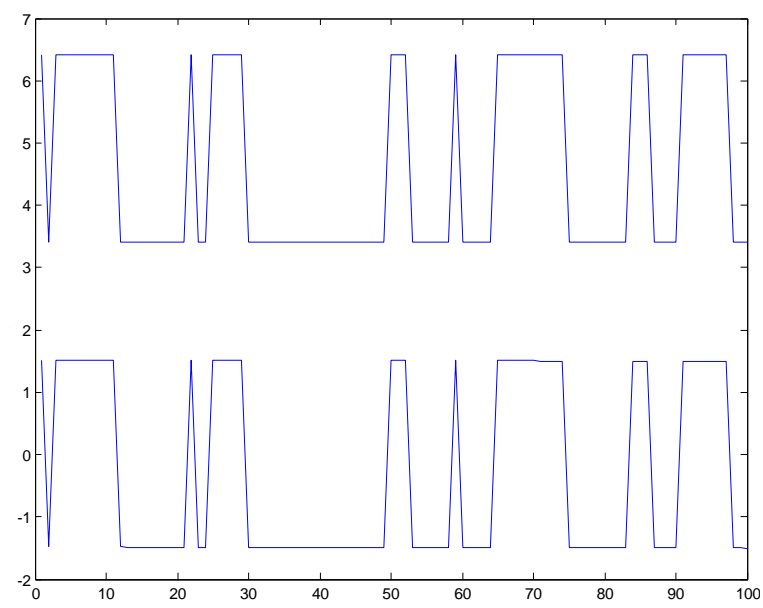

Fig. 5. DC level correction

\subsection{Peak Shaving}

At first, it is necessary to recognize the system dynamics based on input and output data. It is important to smooth the spikes and to apply peak shaving. These spikes are because of a little inaccuracy of sensors or some impedance mismatch and power dissipation in data acquisition card that causes a numerical fault in data representation [34, 35]. Whereas the high energy, interferes the model parameters estimation and its validation. By using a third order Butterworth filter, the filtered data were obtained. The filtered output for dryer is shown in figure 6. In comparison to [4], the results depicted in figure 6 are more satisfactory for implementation [33].

The correlation analysis is used to obtain the weight and correlation dynamics between input and output data [5]. The similarity of two signals will be measured in correlation analysis. In this analysis, the correlation order of two signals is measurable. These contexts for this system could be written as the following equations:

$$
\begin{aligned}
& \Phi_{\mathrm{u}, \mathrm{y}}(\tau)=\mathrm{E}\{\mathrm{u}(\mathrm{t}) \cdot \mathrm{y}(\mathrm{t}-\tau)\} \\
& =\lim _{\mathrm{N} \rightarrow \infty} \frac{1}{\mathrm{~N}} \sum_{\mathrm{k}=1}^{\mathrm{N}} \mathrm{u}(\mathrm{k}) \cdot \mathrm{y}(\mathrm{k}-\tau)
\end{aligned}
$$

where $\Phi_{\mathrm{u}, \mathrm{y}}(\tau)$ is the correlation between $\mathrm{u}$ and $\mathrm{y}$.

$$
\begin{aligned}
& \Phi_{\mathrm{u}, \mathrm{u}}(\tau)=\mathrm{E}\{\mathrm{u}(\mathrm{t}) \cdot \mathrm{u}(\mathrm{t}-\tau)\} \\
& =\lim _{\mathrm{N} \rightarrow \infty} \frac{1}{\mathrm{~N}} \sum_{\mathrm{k}=1}^{\mathrm{N}} \mathrm{u}(\mathrm{k}) \cdot \mathrm{u}(\mathrm{k}-\tau)
\end{aligned}
$$


where $\Phi_{\mathrm{u}, \mathrm{u}}(\tau)$ is the autocorrelation of $\mathrm{u}$.

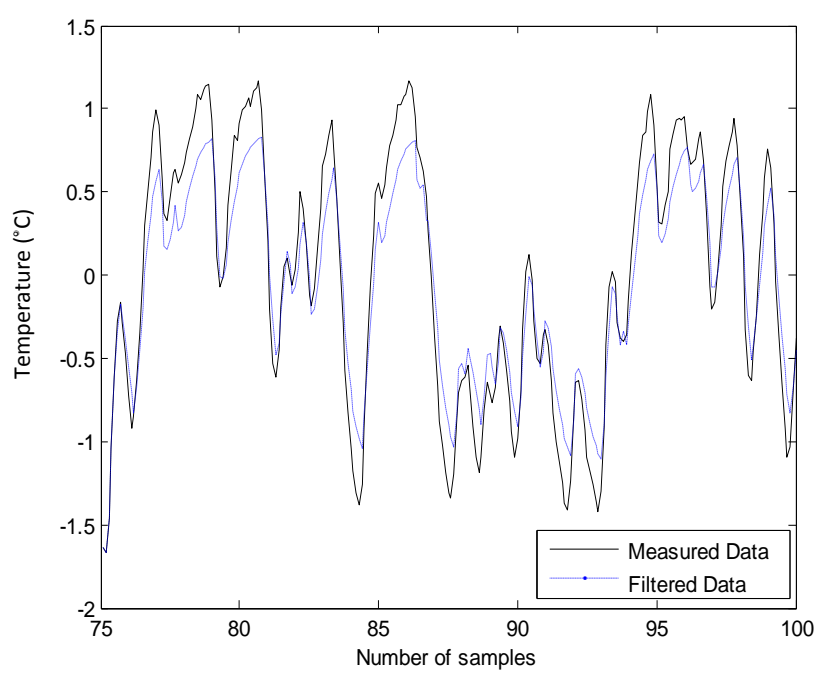

Fig. 6. Measured data and filtered data

\section{LINEAR MODELING WITH ARX MODEL}

The Autoregressive with exogenous input (ARX) model (Figure 7) is an extended autoregressive (AR) model which is represented in equation 3 .

$\mathrm{y}(\mathrm{k})=\frac{\mathrm{B}(\mathrm{q})}{\mathrm{A}(\mathrm{q})} \mathrm{u}(\mathrm{k})+\frac{1}{\mathrm{~A}(\mathrm{q})} \mathrm{v}(\mathrm{k})$

According to figure 7, one of the ARX characteristics is that the disturbance, i.e., the white noise $\mathrm{v}(\mathrm{k})$, is assumed to enter the process before the denominator dynamics A (q) . This fact can be expressed in another way by saying that the ARX model has a noise model of $1 /$ A (q) $[1,31]$.

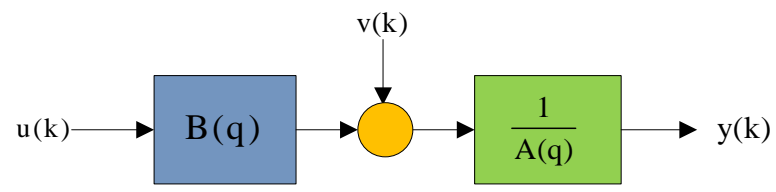

Fig. 7. Diagram of the linear model based on autoregressive with exogenous input

Let the process be described by an nth order difference equation:

$\mathrm{y}(\mathrm{t})+\mathrm{a}_{1} \mathrm{y}(\mathrm{t}-1)+\ldots+\mathrm{a}_{\mathrm{n}} \mathrm{y}(\mathrm{t}-\mathrm{n})$

$=\mathrm{b}_{1} \mathrm{u}(\mathrm{t}-1)+\ldots+\mathrm{b}_{\mathrm{n}} \mathrm{u}(\mathrm{t}-\mathrm{n})$

the transfer function of the process is given by:

$G(q)=\frac{B(q)}{A(q)}$

where

$$
\begin{aligned}
& A(q)=1+a_{1} q^{-1}+\cdots+a_{n} q^{-n} \\
& B(q)=1+b_{1} q^{-1}+\cdots+b_{n} q^{-n}
\end{aligned}
$$

Figure 8 shows the block diagram of error generation in transfer operator estimation using the ARX method [1].

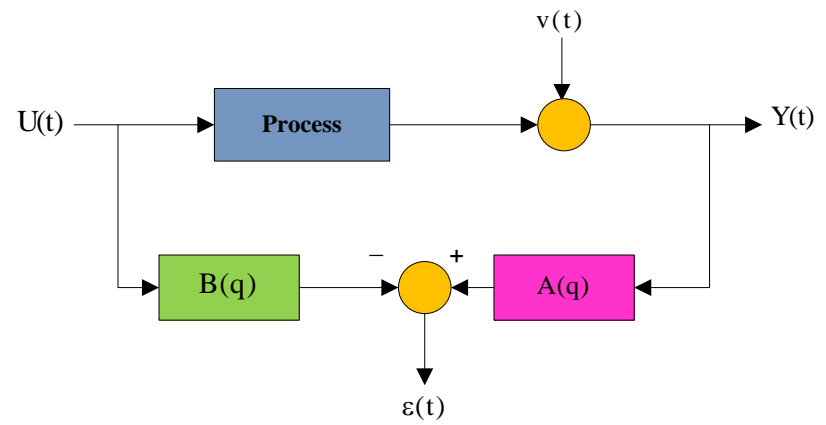

Fig. 8. Error generation in ARX method

The result of simulation with ARX model is shown in figure 9.

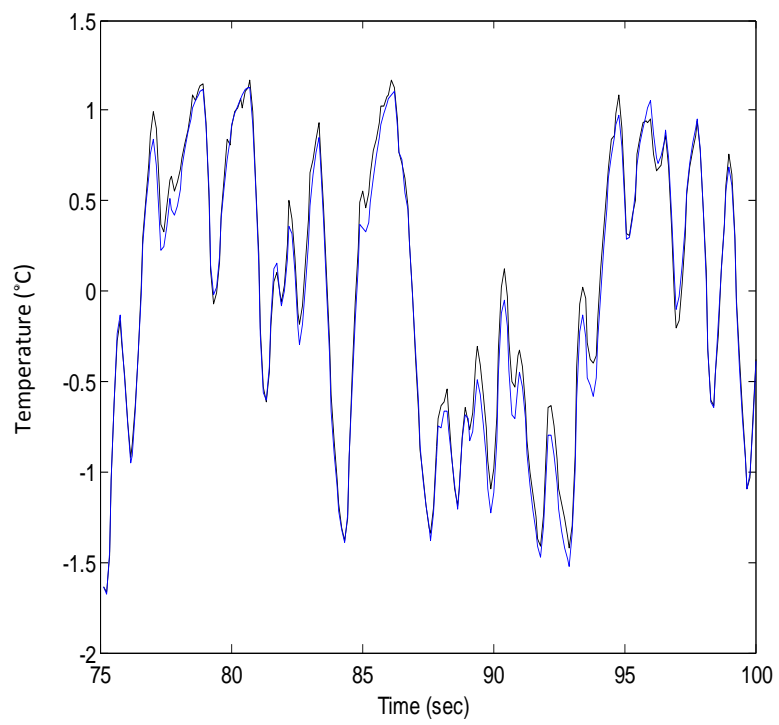

Fig. 9. Simulated model for system with ARX method

According to figure 9, it can be seen that ARX gives a strong fit and it confirms above theories. So as a conclusion, in analysis, ARX is a good model to control the system. The error for output temperature is shown in figure 10 where the RMS value of error for this model amounts to $4.6 \%$. 


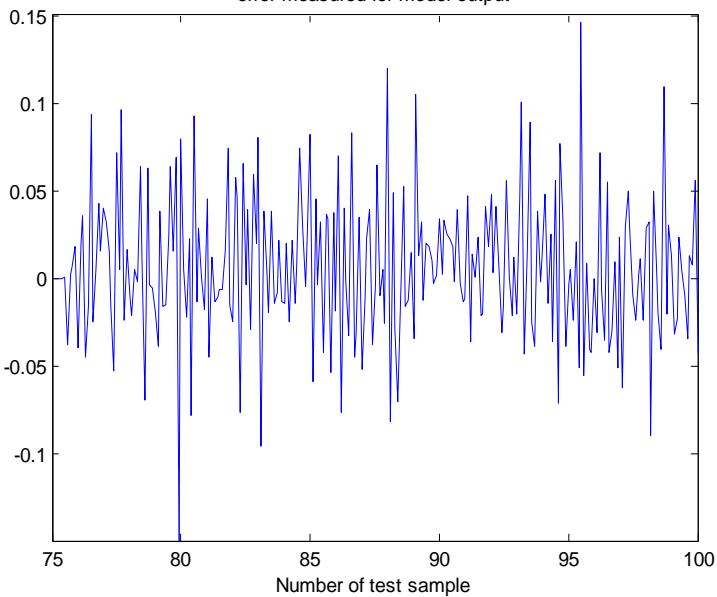

Fig. 10. Error for output temperature

\section{LINEAR MODELING WITH BOX- JENKINS MODEL}

In this model the real system is assumed like below:

$$
y(t)=\frac{B(q)}{F(q)} u(t)+\frac{C(q)}{D(q)} e(t)
$$

where

$$
\begin{aligned}
& F(q)=1+a_{1} q^{-1}+\cdots+a_{n} q^{-n} \\
& B(q)=b_{1} q^{-1}+\cdots+b_{n} q^{-n} \\
& C(q)=1+c_{1} q^{-1}+\cdots+c_{n} q^{-n} \\
& D(q)=1+d_{1} q^{-1}+\cdots+d_{n} q^{-n}
\end{aligned}
$$

This structure has been introduced by Box-Jenkins (BJ) in 1970 [3]. The predictor for this model is:

$$
\frac{d y_{t}}{d \theta}=\frac{D(q) B(q)}{C(q) F(q)} u(t)+\left[1-\frac{D(q)}{C(q)}\right] y(t)
$$

The parameters of this model which are valuable for dryer system will be obtained with the following equation:

$$
\mathrm{V}_{\mathrm{BJ}}=\frac{1}{\mathrm{~N}} \sum_{\mathrm{t}=1}^{\mathrm{N}}\left[\frac{\mathrm{D}(\mathrm{q})}{\mathrm{C}(\mathrm{q})}\left\{\mathrm{y}(\mathrm{t})-\frac{\mathrm{B}(\mathrm{q})}{\mathrm{F}(\mathrm{q})} \mathrm{u}(\mathrm{t})\right\}\right]^{2}
$$

Figure 11 shows the block diagram of Box-Jenkins (BJ) model. The main advantage of the $\mathrm{BJ}$ model is giving a better estimation for the closed-loop models [31]. On the other hand, its implementation is a challenging task. Error generation procedure in $\mathrm{BJ}$ model is shown in figure 12 .

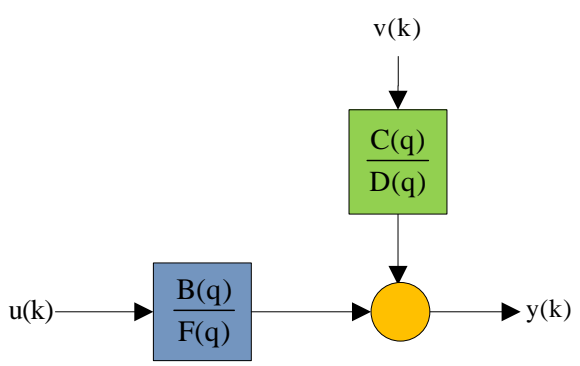

Fig. 11. Block diagram of Box-Jenkins model

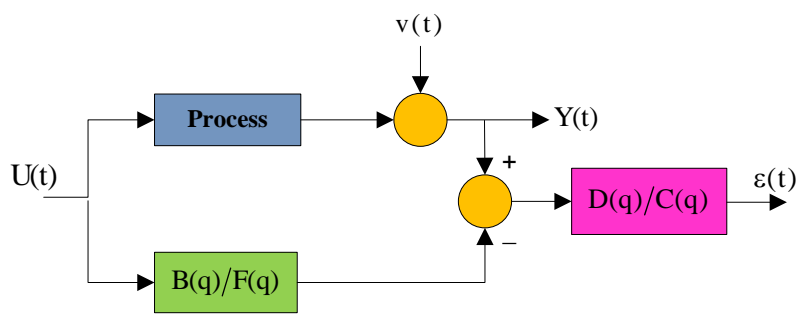

Fig. 12. Block diagram of error generation in $\mathrm{BJ}$ model

The result of simulation with $\mathrm{BJ}$ model is shown in figure 13 . The error for output temperature is shown in figure 14 where the RMS value of error for BJ model amounts to $3.1 \%$.

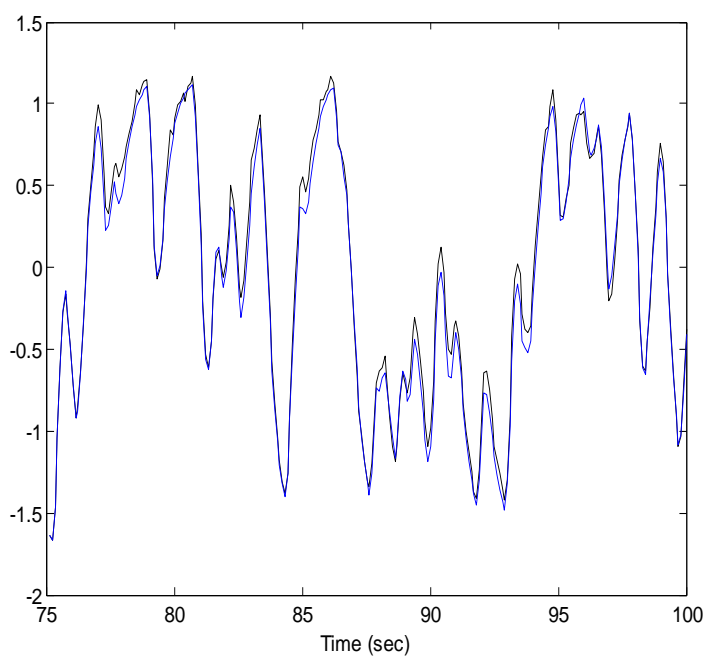

Fig. 13. Simulated model for system with BJ method

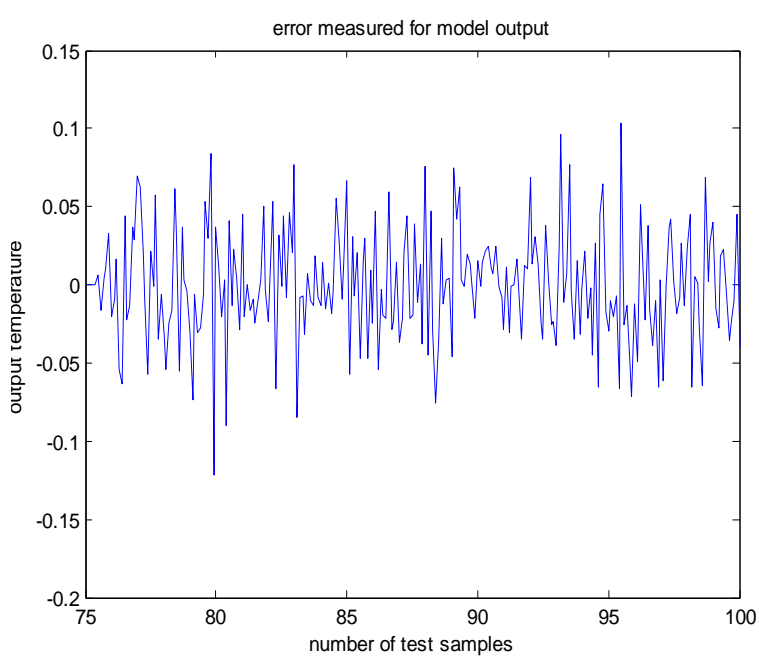

Fig. 14. Error for output temperature 


\section{NONLINEAR MODELING WITH MLP NETWORK}

Next, the Multi Layer Perceptron (MLP) network with Levenberg-Marquadt (LM) learning algorithm has been used for modeling the dryer system. The Levenberg-Marquardt algorithm, which was independently developed by Kenneth Levenberg and Donald Marquardt, provides a numerical solution to the problem of minimizing a nonlinear function. It is fast and has stable convergence. In the artificial neuralnetworks field, this algorithm is suitable for training smalland medium-sized problems [22]. The Levenberg-Marquardt algorithm blends the steepest descent method and the GaussNewton algorithm. Fortunately, it inherits the speed advantage of the Gauss-Newton algorithm and the stability of the steepest descent method. It's more robust than the GaussNewton algorithm, because in many cases it can converge well even if the error surface is much more complex than the quadratic situation. Although the Levenberg-Marquardt algorithm tends to be a bit slower than Gauss-Newton algorithm (in convergent situation), it converges much faster than the steepest descent method. The basic idea of the Levenberg-Marquardt algorithm is that it performs a combined training process: around the area with complex curvature, the Levenberg-Marquardt algorithm switches to the steepest descent algorithm, until the local curvature is proper to make a quadratic approximation; then it approximately becomes the Gauss-Newton algorithm, which can speed up the convergence significantly [23-25, 28, 29]. In order to make sure that the approximated Hessian matrix $\mathrm{J}^{\mathrm{T}} \mathrm{J}$ is invertible, Levenberg-Marquardt algorithm introduces another approximation to Hessian matrix as shown in Equation 11.

$\mathrm{H}=\mathrm{J}^{\mathrm{T}} \mathrm{J}+\mu \mathrm{I}$

where

$\boldsymbol{\mu}$ is always positive, called combination coefficient

I is the identity matrix

From Equation 11, one may notice that the elements on the main diagonal of the approximated Hessian matrix will be larger than zero. Therefore, with this approximation (Equation 11 ), it can be sure that matrix $\mathrm{H}$ is always invertible. As a point of view, the updated rule of Guass-Newton algorithm is presented as Equation 12

$\mathrm{w}_{\mathrm{k}+1}=\mathrm{w}_{\mathrm{k}}-\left(\mathrm{J}_{\mathrm{k}}^{\mathrm{T}} \mathrm{J}_{\mathrm{k}}\right)^{-1} \mathrm{~J}_{\mathrm{k}} \mathrm{e}_{\mathrm{k}}$

By combining Equations 11 and 12, the update rule of Levenberg-Marquardt algorithm can be presented as Equation $13[26,27]$.

$\mathrm{w}_{\mathrm{k}+1}=\mathrm{w}_{\mathrm{k}}-\left(\mathrm{J}_{\mathrm{k}}^{\mathrm{T}} \mathrm{J}_{\mathrm{k}}+\mu \mathrm{I}\right)^{-1} \mathrm{~J}_{\mathrm{k}} \mathrm{e}_{\mathrm{k}}$

The MLP is the most applicable neural network. This network is able to perform a nonlinear mapping with the correct choice of neuron weights and transfer function bias [20,21]. The MLP network structure is shown in figure 15.

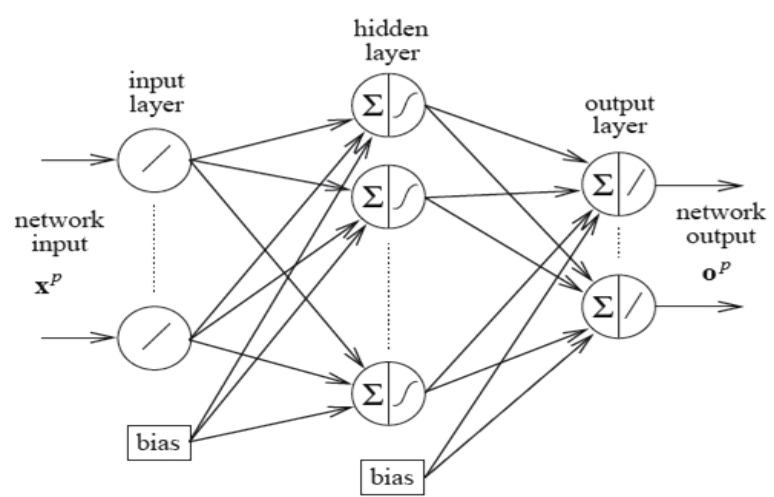

Fig. 15. Structure of one layer perceptron

This network contains two hidden layers and an output layer. The network inputs take the weights and biases, and then go to the nonlinear part. This part involves a transfer function that has been assumed as $\mathrm{y}=\tanh (\mathrm{x})$. The Levenberg Marquadt learning algorithm has been used for system identification. Figure 16 and figure 17 show the result of simulation with a constant number of neurons. The epochs are the number of iteration to train the network. According to figure 16 , the system performance amounts to 0.0443084 and Goal $=0$.

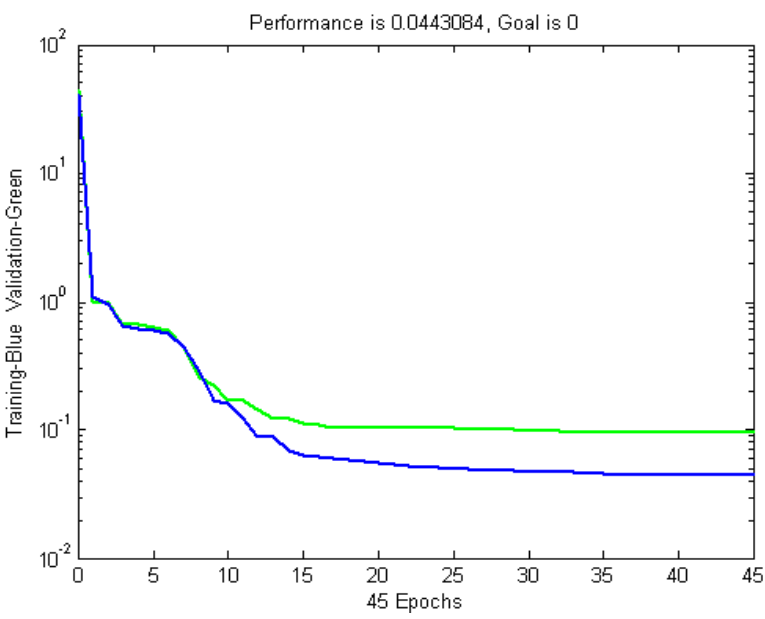

Fig. 16. Training function for test and train data

\subsection{Effect of Increasing Number of Neurons in the Hidden Layer}

By increasing the number of neurons, a better fitness could be met. On the other hand, increasing the neurons more than optimal case, results overtraining in system and this is inadequate. 


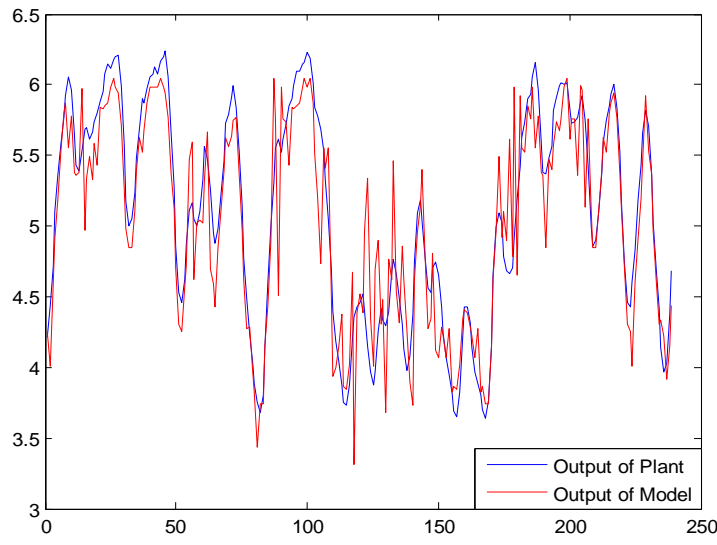

Fig. 17. Plant output based on the LM learning algorithm

For each number of neurons, the weights and biases in the first layer have been chosen between 0 and 1 randomly to prevent overtraining. This allows the network to be trained also within the negative data in the output.

Initially the number of neurons was 9 in the first layer and 9 in the second layer. In order to achieve an optimal training the number of neurons were modified in hidden layer from 9 to 12 in the first layer and 9 to 15 in the second layer, that they are optimal case for training. The result of simulation in this case is shown in figure 18 and figure 19.

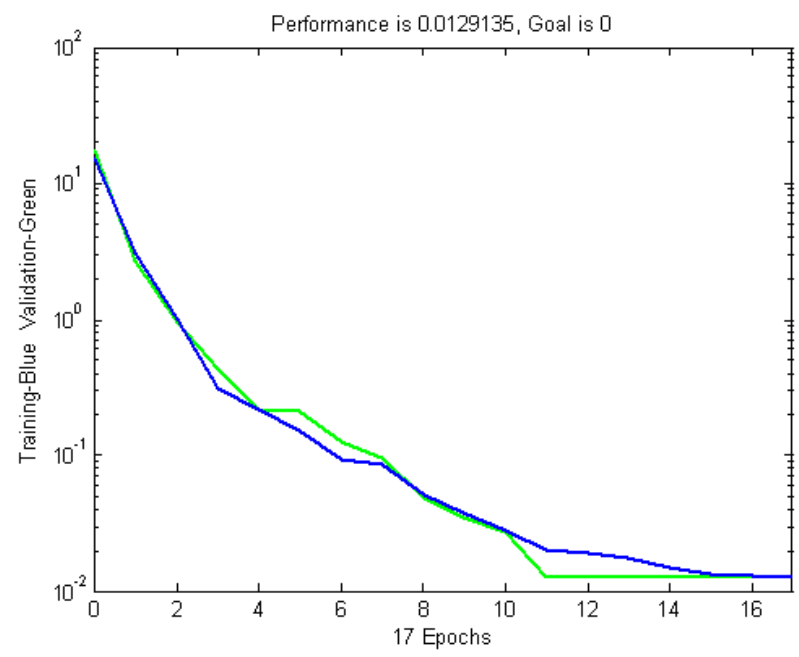

Fig.18. Training function for test and train data

Figure 20 shows the block diagram of error generation in MLP algorithm which the calculation of error is mentioned in Equation 14 [1, 31]. Figure 21 shows the calculated error in MLP learning algorithm, which results in $1.85 \%$ RMS value introducing the error between input and output of plant in this algorithm.

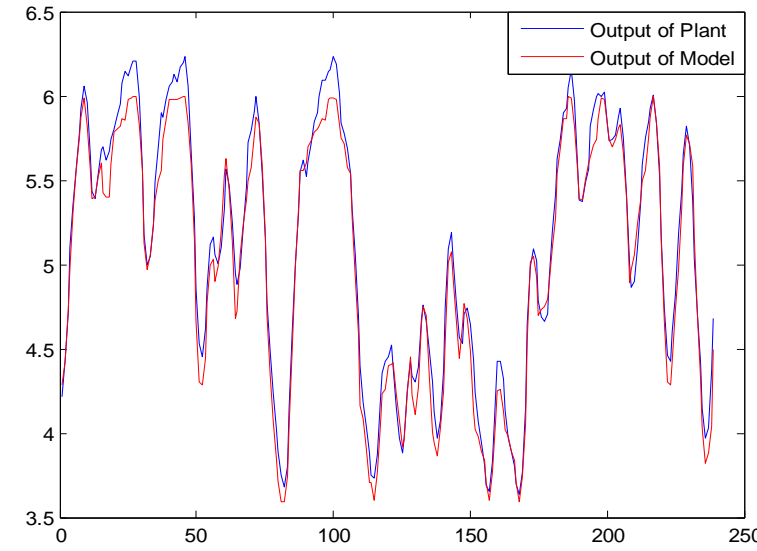

Fig. 19. Plant output based on LM with increasing number of neurons in the hidden layer

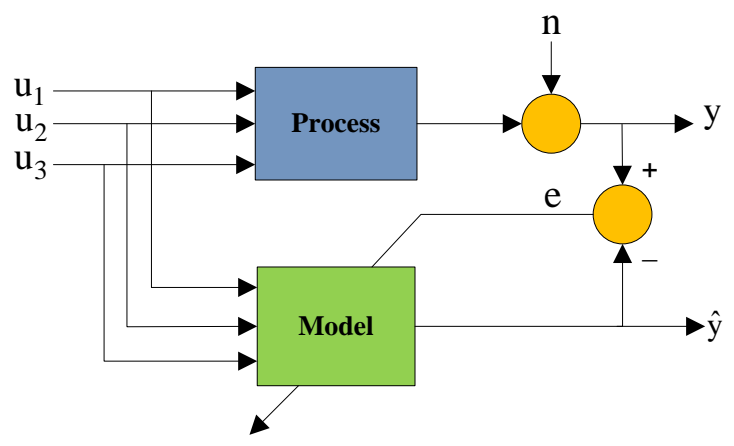

Fig. 20. Block diagram of error generation in MLP algorithm

$$
\begin{aligned}
& y=u \cdot P+n \\
& \hat{y}=u \cdot M \\
& e=y-\hat{y}
\end{aligned}
$$

where $\mathrm{P}$ and $\mathrm{M}$ are the transfer function of Process and Model, respectively.

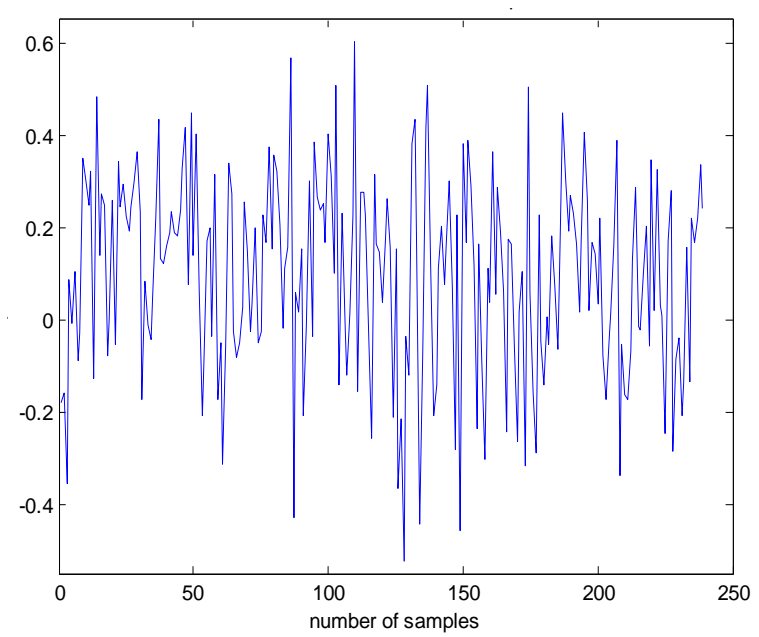

Fig. 21. Error measured for model output based on LM learning algorithm 


\section{CONCLUSIONS}

A theoretical comparison between two linear models for a temperature control dryer system was presented. According to environment conditions and system configuration, using the fundamental identification methods could not comprise reasonable results for identification task. So, as a novel method, the identification techniques based on system configuration and input-output relation obtained from data acquisition was formulized. In nonlinear identification, the output of dryer system have been trained, based on its input and output with a two-layer MLP network and the LM learning algorithm. By changing the number of neurons, in the worst case, the RMS value for test data was about $1.85 \%$ that means the system identification is around the operation point. Based on results it could be concluded that these models can be used to design adaptive or robust controllers and also, the Box-Jenkins model presented better results for this application compared to the ARX model.

\section{ACKNOWLEDGMENTS}

The author would like to thank those engineers and technical staffs who were working on the plant providing some facilities during this project.

\section{REFERENCES}

[1] Nelles, O., 2001, Nonlinear System Identification-From Classical Approaches to Neural Networks and Fuzzy Model, Springer-Vetlag, Berlin Heidelberg, New York.

[2] Nelles, O., Fink, A. and Isermann, 2000, Local Linear Model Trees (LOLIMAT), toolbox for nonlinear system identification, IFAC.

[3] Billings, S. A. and Fakhouri, S. Y., 1982, Identification of systems containing linear dynamic and static nonlinear elements, Automatica, pp.15-26.

[4] Oliveira, G. H. C., Da Rosa, A., Campello, J.G.B. R., Machado, B. J., and Amaral, W. C., 2012, An introduction to models based on Laguerre, Kautz and other related orthonormal functions - Part II: non-linear models, Int. J. of Modelling, Identification and Control (IJMIC), Vol. 16, No.1, pp.1 - 14.

[5] Syariful S., Sh., and XiaoQi, Ch., 2012, Identification of an unmanned helicopter system using optimised neural network structure, Int. J. of Modelling, Identification and Control (IJMIC), Vol.17, No.3, pp.223 - 241.

[6] Wei, Ch., and Junfei, Q., 2012, Nonlinear system identification and fault detection method using RBF neural networks with set membership estimation, Int. J. of Modelling, Identification and Control (IJMIC).

[7] Ben-Zvi, A., 17-19 July 2010, Modeling and parameter estimation for complex systems, 2nd International conference of Modelling, Identification and Control (ICMIC), Okayama, Japan.

[8] Stoyan, V. A., 2003, Identification Problem for MatrixIntegrating Systems, Cybernetics and Systems Analysis, Volume 39, Number 5, pp. 753-764(12).

[9] Dayal, B. S., and MacGregor, J. F., 1997, Multi-output process identification, Journal of Process Control, Volume 7, Number 4, pp. 269-282(14).

[10] Taarit, I., Belkoura, K., Lotfi, K., Mekki, R., 2011, A fast identification algorithm for systems with delayed inputs, International Journal of Systems Science, Volume 42, Number 3, pp. 449-456(8).
[11] Alfi, A., Fateh, M., 2011, Identification of nonlinear systems using modified particle swarm optimisation: a hydraulic suspension system, Vehicle System Dynamics, Volume 49, Number 6, pp. 871887(17).

[12] Rudenko, O. G., Bessonov, A.A., 2003, Real Time Identification of Nonlinear Time Varying Systems Using Radial Basis Function Network, Cybernetics and Systems Analysis, Volume 39, Number 6, pp. 927-934(8).

[13] Ljung, L., 1999, System Identification, John Wiley \& Sons.

[14] Narendra, K. S., and Parthasarathy, K., 1990, Identification and Control of Dynamical Systems Using Neural Networks, IEEE Transactions on Neural Networks, Vol. 1, No. 1

[15] Sjoberg, J., Zhang, Q., Ljung, L., Benveniste, A., Delyon, B., Glorennec, P. Y., Hjalmarsson, H., and Juditsky, A., 1995, Nonlinear black-box modeling in system identification: a unified overview, Automatica, Vol. 31, No. 12, pp. 1691-1724.

[16] Billings, S. A., and Fakhouri, S. Y., 1982, Identification of systems containing linear dynamic and static nonlinear elements, Automatica, Vol. 18, No. 1, pp. 15-26.

[17] Emara-Shabaik, H. E., 1996, Nonlinear systems modeling \& identification using higher order statistics/polyspectra, Control and Dynamic Systems, Vol. 76, pp. 289-322.

[18] Bongard, J. C., and Lipson, H., 2005, Nonlinear System Identification Using Coevolution of Models and Tests, IEEE Transactions on Evolutionary Computation, Vol. 9, No. 4.

[19] Juang, J-G, 2005, Nonlinear System Identification by Evolutionary Computation and Recursive Estimation Method, American Control Conference, Portland, OR, USA.

[20] Aleksander, I., and Moton, H., 1995, An Introduction to Neural Computing, Internat. Thomson Computer Press.

[21] Ash, T., and Cottrell, G., 1995, Topology-modifying neural network algorithms, The Handbook of Brain Theory and Neural Networks, Michael A. Arbib (ed.), MIT Press, Cambridge MA.

[22] Baldi, P., and Hornik, K., 1989, Neural Networks and Principal Components Analysis: Learning from examples without local minima, Neural Networks, Vol. 2, No. 53.

[23] Hagan, M. T., Demuth, H. B., and Beale, M. H., 2002, Neural Network Design, Vol. 10, Campus Publishing Service, Colorado University Bookstone.

[24] Rosenblatt, F., Principles of Neurodynamics: Perceptrons and the Theory of Brain Mechanisms, Spartan Press, Washington, DC, 1961.

[25] Riedmiller, M., and Braun, H., 1993, A Direct Adaptive Method for Faster Backpropagation Learning: The RPROP Algorithm, Proceedings of the IEEE International Conference on Neural Networks.

[26] Vogl, T. P., Mangis, J. K, Rigler, A. K., Zink, W. T., and Alkon, D. L. 1988, Accelerating the convergence of the 
backpropagation method, Biological Cybernetics, Vol. 59 , pp. 256-264.

[27] Hagan, M. T., and Menhaj, M., 1994, Training feedforward networks with the Marquardt algorithm, IEEE Transactions on Neural Networks, Vol. 5, No. 6, pp. 989-993.

[28] Kohonen, T., 1988, Self-organization and Associative Memory, $2^{\text {nd }}$ Edition, Berlin: Springer-Verlag.

[29] Kohonen, T., 2001, Self-Organizing Maps, Springer.

[30] Ljung, L., 1998, Nonlinear System Identification: Theory for the User, Pearson Education.

[31] Eykhoff, P., 1974, System Identification: parameter and state estimation, Wiley-Interscience, Mathematics.
[32] Wang, L., and Garnier, H., 2012, System Identification, Environmetric Modeling and Control, Springer.

[33] Tulleken, H.J.A.F., Generalized binary noise test-signal concept for improved identification-experiment design, Automatica, vol. 26, No. 1, pp. 34-49, 1990.

[34] Juang, J-N., 1994, Applied System Identification, Prentice-Hall, Inc. Upper Saddle River, NJ, USA.

[35] Billings S.A, and Voon W.S.F., 1986, Correlation based validity tests for nonlinear models, International journal of Control, Vol. 44, No. 1, pp. 235-244.

[36] Billings S.A, and Voon W.S.F., 1987, Piecewise linear identification of non-linear systems, International journal of Control, Vol. 46, No. 1, pp. 215-235. 